\title{
Repeated Detoxification of Alcohol-Dependent Patients Impairs Brain Mechanisms of Behavioural Control Important in Resisting Relapse
}

\author{
Theodora Duka • David N. Stephens
}

Published online: 8 January 2014

(C) Springer International Publishing AG 2014

\begin{abstract}
Alcohol abuse is frequently characterised by cycles of heavy drinking, detoxification, and relapse. We review evidence that multiple detoxifications are associated with impaired ability to control reward seeking, and with exaggerated responses to negative emotional stimuli. Under conditions of incentive conflict and in intra-extra dimensional shift and reversal tasks, deficits are found that are consistent with impaired executive control of behaviour by prefrontal cortical mechanisms. Correspondingly, alcoholics who have undergone multiple detoxifications show loss of grey matter in prefrontal regions associated with accurate performance of these tasks, the extent correlating with numbers of detoxifications. The ability to respond appropriately to certain emotional stimuli (e.g., fearful faces) is also impaired following multiple detoxifications. Such impairments are associated with reduced connectivity between insula and prefrontal areas but increased connectivity between insula and subcortical regions (colliculus), and between amygdala and other subcortical regions (bed nucleus of stria terminalis, BNST). Such changes may increase vulnerability to stress-induced relapse, and disrupt social abilities, contributing to social isolation.
\end{abstract}

Keywords Alcoholism · Withdrawal · Conditioning · Executive function - fMRI - Functional connectivity - BNST Colliculus $\cdot$ Insula $\cdot \mathrm{vmPFC} \cdot$ Incentive conflict task . Emotional Facial Expression (EFE) recognition

T. Duka $\cdot$ D. N. Stephens

Behavioural and Clinical Neuroscience, School of Psychology,

University of Sussex, Brighton, UK

T. Duka $(\triangle)$

School of Psychology, University of Sussex, Falmer, Brighton BN1 $9 \mathrm{QH}, \mathrm{UK}$

e-mail: t.duka@sussex.ac.uk

\section{Introduction}

Alcohol Use Disorder (AUD; alcohol abuse and dependence) is a chronic relapsing disorder characterised by compulsive drinking. Among Americans 15-54 years old, about one in seven $(14 \%)$ has a history of alcohol dependence [3]. In the Europe Union, AUD affects an estimated $5.4 \%$ of all men, and $1.5 \%$ of all women, between the ages of 18 and 64 [78]. AUD has severe consequences for the life of dependent individuals, their families and friends, work colleagues and customers, as well as for the health and social services, the police and the general public. Even approved treatments ${ }^{1}$ such as disulfiram [44], naltrexone [2], acamprosate [59], the newly introduced nalmefene [58] or cognitive/behavioural treatments are of limited efficacy and may be effective in controlling drinking in only subpopulations of misusers. One consequence is that a high proportion of alcohol-dependent individuals relapse after detoxification and by repeated attempts at detoxification will undergo a phenomenon known as withdrawal kindling [36], which, as well as increasing the risk of detoxification for seizures $[6,16,53]$, makes each subsequent effort to abstain increasingly difficult. Understanding the mechanisms by which relapse occurs would facilitate the identification of useful biomarkers and would lead to new intervention strategies. The purpose of this review is to describe possible cognitive and emotional processes (and their underlying brain mechanisms), which weaken as alcohol dependence and number of detoxifications progress, and may contribute to relapse (see Fig. 1).

\footnotetext{
${ }^{1}$ Approved medications for the treatment of alcohol dependence by both the Food and Drug Administration (FDA) and the European Medicines Agency (EMA) are disulfiram, naltrexone and acamprosate. EMA has also approved nalmefene for the reduction in heavy drinking, whereas FDA has approved in addition the depot form of naltrexone.
} 


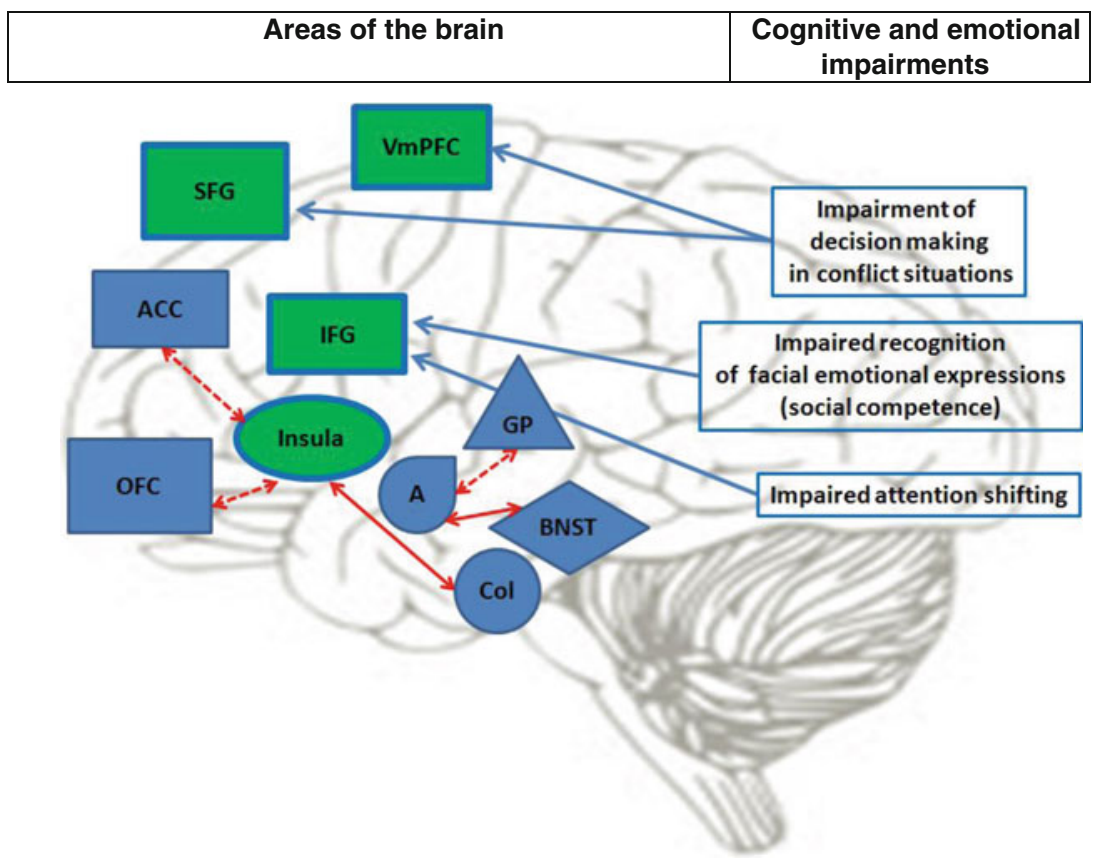

Fig. 1 A model of withdrawal kindling and its consequences for relapse. Findings in alcoholic patients with two or more previous detoxifications compared with controls or patients with one or no detoxifications: Prefrontal cortical areas with reduced grey matter (green). Cognitive and emotional impairments on tasks of emotional conflict associated with motivation (ICT), social competence (EFE task), and cognitive flexibility (ID/ED shift and reversal task) and the areas of the brain with reduced grey matter associated with these impairments (blue arrows indicate the relationships). Reduced functional neuronal connectivity between insula and prefrontal cortical areas as well as between amygdala and globus pallidus (red broken arrows indicate reduced connectivity). Increased functional connectivity between insula and a colliculus cluster as well

\section{Top-Down and Bottom-Up Processes Involved in Relapse}

AUD may be ascribed to two basic processes, increased urges to take drugs and reduced ability to control drinking. These processes are sometimes referred to respectively as 'bottomup' and 'top-down', reflecting the view that urges to drink are largely controlled by subcortical mechanisms, whereas control of drinking is exercised by cortical processes that monitor excessive behaviours. Similar ideas have been developed in the context of other drug use disorders [35••]. The major topdown process is inhibitory control. Inhibitory control, necessary for self-regulation, is linked to executive function capacity, as individuals with low executive capacity or damage to brain substrates subserving executive function display reduced ability to self-regulate and a greater susceptibility to stimulus-driven behaviours [23,42,92]. It is not surprising, therefore, that executive deficits have been associated with alcoholism [33], a disorder characterised by the lack of ability to self-regulate drinking [7]. The ability to disengage attention efficiently from stimuli which are irrelevant to the present task or are in opposition to the individual's current goals, as between amygdala and BNST (red arrows indicate increased connectivity). The model suggests that both a top-down behavioural control insufficiency and a bottom-up increased emotional input constitute the consequences of multiple detoxifications. Thus, not only responsiveness to stressors or to alcohol-related cues is increased, but the emotional regulation and behavioural inhibition systems are weakened. $A$ amygdala, $A C C$ anterior cingulate cortex, $B N S T$ bed nucleus of stria terminalis, $\mathrm{Col}$ colliculus, $E F E$ emotional facial expression, $G P$ globus pallidus, $I C T$ incentive conflict task, $I D / E D$ intradimensional/ extradimensional, $I F G$ inferior frontal gyrus, $O F C$ orbitofrontal cortex, $S F G$ superior frontal gyrus, $V m P F C$ ventromedial prefrontal cortex. Position of named structures for illustrative purposes only

represents a critical dimension of all self-regulatory behaviour $[22,60]$. This ability depends on inhibitory cognitive processes, working memory capacity, and the ability to shift attention from previously relevant (but now irrelevant) stimuli (e.g., alcohol cues) to currently relevant factors (e.g., awareness of drinking consequences).

As AUD progresses, alcohol-associated stimuli become more salient and grab attention more readily (attentional bias; e.g., $[32,40,89])$, resulting in an increased urge to drink. In alcoholic patients, such alcohol-associated attentional bias predicts relapse rates and treatment outcomes (e.g., [17]). Such alcohol-related stimuli, with strong emotional and motivational salience, act in a bottom-up fashion (e.g., highly rewarding stimuli; $[1 \bullet \bullet])$ to out-compete other thoughts or activities, driving the organism to relapse. Recent functional magnetic resonance imaging (fMRI) studies have provided evidence for the involvement of brain substrates associated with cognitive control in attentional bias to drug-related stimuli $[41,57,70]$. Thus, as dependency on the drug progresses, relapse after efforts at abstinence becomes increasingly likely as distinct places, people, and paraphernalia associated with 
the reward offered by the drug trigger an intense motivation within the individual to consume the drug.

Within this context, inhibitory control represents an important cognitive domain modulating the translation of desire to drink into alcohol consumption, and such weakening of inhibitory control may lead to drug use disorders $[31,38,93]$. Strengthening inhibitory control may thus prove the best strategy to prevent relapse [15].

\section{Relapse as a Cause of Emotional and Cognitive Consequences}

Among alcoholics, a typical pattern consists of problem drinking, treatment involving detoxification, a period of abstinence (which may be very short), followed by relapse. Such cycles may be repeated many times during an alcoholic career, each successive withdrawal episode being associated with increased risk of seizures and other withdrawal symptoms $[16,53]$. Over the last decade, evidence has accumulated to show that patients who have undergone repeated episodes of withdrawal also show altered affect, as well as significant deterioration of cognitive abilities, when compared with patients with fewer withdrawals (e.g., [25,26]). The pattern of repeated episodes of heavy drinking combined with withdrawal also occurs, perhaps in a milder form, in another type of alcohol misuse, binge drinking, and related impairments have also been shown in young binge drinkers (BDs; e.g., $[20,64,69,84,91])$.

Although a number of investigators had pointed out that repeated episodes of detoxification increases the risk of withdrawal seizures, an important extension of those reports came with the discovery [52] of the differential response of alcoholics with two or more detoxifications compared with those with only one, with regard to their sensitivity to anxiety evoked by the noradrenergic $\alpha 2$ agonist, yohimbine. More recently, we reported that within a group of mildly dependent alcoholic inpatients more than one week into detoxification, patients with a history of two or more previous detoxifications showed higher anger scores than patients with fewer detoxifications, and made more errors in an emotional Stroop test using words with a negative relationship to alcohol abuse (e.g., hangover; vomit); these tests suggested an increased attentional bias to aversive events associated with alcohol [25]. The same patients, relative to alcoholics with fewer detoxifications, also showed deficits in psychometric tasks sensitive to dysfunction of prefrontal areas [26]. Studies in animals have implicated the amygdala [76] and its accessory structures, including inferior colliculus [63], in seizure predisposition induced by repeated withdrawals $[11,85]$. Consistent with these ideas, recognition of facial expression of fear, which is known to be disrupted in amygdala-damaged patients, was also found to be impaired in alcoholic patients
$[49,50,90]$. Furthermore, alcoholic patients showed a correlation between the number of detoxifications they had undergone and the extent of impaired recognition of fear expression in the faces of others [90].

In keeping with facilitated transmission in amygdala, rats that have previously undergone several cycles of chronic alcohol intake and withdrawal show evidence of enhanced anxiety during acute withdrawal in some unconditioned tests [74]. In addition, several weeks following such a regimen of alcohol treatment and withdrawal, rats are impaired in fear conditioning to a tone or light stimulus paired with footshock $[80,85]$, possibly as a consequence of reduced synaptic plasticity within the amygdala [87•]. Similarly, alcoholic patients compared with controls are impaired in a conditioned startle response [86].

Taking all these findings together, it becomes clear that alcoholic patients with multiple detoxifications, even as few as two, present with more emotional and cognitive impairments than alcoholics with fewer detoxifications, or their control counterparts (for reviews see $[24,86]$ ). Nevertheless, the potential impact of these impairments leading to relapse and the brain mechanisms underlying these events in humans has only recently been the focus of research.

\section{Inhibitory Control and Incentive Conflict, Brain Mechanisms}

As discussed above, recent theories of drug and alcohol abuse [45] emphasise the importance of (i) bottom-up incentive processes in initiating drinking, often as a consequence of exposure to environmental events (cues) that have become conditioned to the drug experience, and of (ii) top-down cortical control of behaviour in the regulation of drug taking. Incentive mechanisms are mediated by brain circuitries including ventral striatum, but are regulated by structures such as orbitofrontal and medial prefrontal cortex [62]. Whether incentive exposure results in drug taking depends on the ability of higher level monitoring functions to interrupt the incentive process, but could also depend on the strengthening of the incentive process as drug use disorder progresses.

While the most prominent current 'theories of addiction' (e.g., [81]) emphasise how drug exposure facilitates bottomup processes, it is increasingly recognised that the brain areas responsible for such higher level functions are themselves sensitive to disruption by long-term drug use. Alcohol abuse, in particular, may impair processes that contribute to impulse control [68], so that, faced with alcohol-related cues, alcoholics are motivated to drink, but the means of controlling drinking are impaired. In the alcoholic patient attempting abstinence, this conflict between the desire to take the drug and the requirement to abstain may be particularly intense, but, in comparison with the wealth of data on bottom-up 
processes, this interaction between incentive and control processes has not been widely studied.

Recently, aspects of the interaction between incentive learning and behavioural control have been studied using a novel task [27••], the incentive conflict task (ICT), a version of negative patterning tasks used in cognitive psychology $[79,94]$. The ICT requires experimental subjects to abstain from responding during presentation of a novel compound stimulus made up of two visual cues that s/he has previously learned to signal reward availability when presented separately. Thus, the task requires the subject to respond for the monetary reward, but to withhold responding under conditions in which an increased size of reward (both positive cues together) might be reasonably anticipated. In other words, ICT engages both bottom-up triggers of reward seeking, and the top-down processes that normally modulate and veto responses to such triggers. We have suggested that the task thus creates a conflict between abstaining and responding for reward similar to that experienced by the patients before relapse, and that the impaired ability of multiple-detoxified patients to perform the task accurately reflects the consequences of detoxification on top-down control of their behaviour.

Alcohol-dependent patients show marked impairments on this task, and, furthermore, as they experience successive detoxifications, and their dependence increases, they become increasingly impaired in withholding their responses in the condition of no reward, suggesting that the process of detoxification may engender brain changes that lead to loss of control $[27 \bullet \bullet]$. Although it is not easy in patients to separate the extent of dependency from numbers of detoxification events, we have found similar deficits in a rodent version of the same task, in rats chronically exposed to alcohol [14••]. Importantly, in this carefully controlled animal study, the number of withdrawal events (detoxifications) determined the extent of the deficit, strongly suggesting that it was the number of withdrawal events, and not simply ethanol exposure that caused the top-down deficit.

The ICT task is not only a behavioural test for alcoholinduced impairments in functions associated with the ability to make appropriate decisions and abstain from seeking reward, but is also a marker of brain function as shown in fMRI [27••]. During performance of the ICT, healthy volunteers showed activation of, among other areas, the supplementary motor area, striatum (including putamen), gyrus rectus, ventromedial prefrontal cortex (vmPFC) and superior frontal gyrus areas implicated in cognitive and emotional processing of reward $[13,28,72]$ and regulatory control over a behavioural response $[34,75]$. The two last areas mentioned showed decreases in grey matter volume in alcoholic patients, which were greater for the patients who had experienced more detoxifications, suggesting that the brain damage is associated with the extent of alcohol dependence, and may be caused by the detoxification experience.
The neural signature of the ICT was distinct from patterns of regional activation evoked by simple reversal of a stimulus from signaling reward to not signaling reward. The ICT signature was also distinct from presentation of the simple reward-predictive stimuli, which resulted in a pattern of activation within a different subregion of orbitofrontal cortex (medial orbitofrontal), as well as insular cortex, consistent with previous research examining stimulus-reward-responserelated learning (e.g., $[12,29,72,88])$. Thus, we are confident in suggesting that the ICT captures a specific function associated with a process of resolving a conflict generated by the need to withhold reward seeking in a context that encouraged reward seeking. Once this cognitive process is complete, the subject has to withhold the behavioural response normally required during reward seeking. Thus, the task involves two separate aspects, a cognitive one for conflict resolution and a behavioural one for withholding of the response; these two processes are uniquely involved in the ICT, can be impaired independently and can be measured by the expectancy ratings of reward outcome and by the response rate in the presence of the compound stimulus, respectively. Patients were impaired both in resolving the conflict and withholding their response. As an imaging biomarker, performance is associated with BOLD signals in vmPFC and prefrontal gyrus areas, reflecting conflict resolution and inhibitory control, respectively.

The involvement of the vmPFC, an area involved in motivational decision-making [8,9], and of superior frontal gyrus, important in behavioural control [34,75], is of particular interest since alcoholic patients showed reliably reduced grey matter volume in these regions. Thus, although performance of incentive conflict was associated with activation of several related brain areas (e.g., supplementary motor area, putamen and gyrus rectus), the behavioural deficit in alcoholic patients may reflect damage to only a subset of these regions, in particular the vmPFC and superior frontal gyrus. Activation of vmPFC is shared with the gambling task [9] which resembles incentive conflict in requiring decision making. It is thus interesting that alcoholic patients with several detoxifications also show impairments in this task [56]. However, the ICT additionally involves conflict generated by the contradictory information carried by the compound stimulus, which might be assumed to predict increase of reward (both positive stimuli together), but actually informs of absence of reward. The cognitive and emotional processes that govern conflict resolution during ICT seem to be associated with the activation seen in vmPFC [27••]. This assumption is further supported by a study [30] that also found that resolution of emotional conflict was associated with activation of an area that included the vmPFC. Prefrontal gyrus activation on the other hand may be more associated with the attentional and executive processes involved in inhibitory control that govern responding to ICT $[34,37,75]$. Alcohol-dependent patients and especially those with multiple detoxifications show damage in these 
areas. Interestingly, Goldstein and colleagues [39], studying the effects of methylphenidate on inhibition of behaviour (using the Stroop task) under conditions of emotional conflict (drug or non-drug cues) in individuals with cocaine use disorder, found that methylphenidate restored performance and also activation of an area that includes vmPFC. Thus, it might be predicted that by restoring function in these areas, drugs such as methylphenidate may help to reinstate control over drinking in relapsed alcoholic patients.

Clearly, alcohol- and detoxification-induced damage is not restricted to the areas identified in the ICT experiments. Alcoholic patients show also more generally impaired cognitive flexibility as measured in an intra-extra dimensional shift and reversal task (IED) that is associated with reduced grey matter volume in a cluster within inferior frontal gyrus and the neighbouring anterior insula (BA47) [unpublished]. The inferior frontal gyrus has been implicated in previous research during cognitive set switching [46], and also when resolving decision conflict during an instrumental learning task [67]. Previous research with alcoholic patients has shown that perseverative responses and perseverative errors in the Wisconsin Card Sorting Test (WCST; an homologous task to IED) also correlated negatively with volume of prefrontal areas (e.g., prefrontal gyrus, an area involved in inhibitory control) [43]. Although grey matter volume was found to be reduced in several prefrontal and frontal areas, the number of detoxifications was negatively correlated ( $\mathrm{r}=-0.47, p=0.017)$ with grey matter volume in inferior frontal gyrus [unpublished], an area involved in inhibitory control, suggesting that decreased inhibitory control due to inferior frontal gyrus damage may support the occurrence of repeated relapses.

\section{Inhibitory Control and Social Competence: Brain Mechanisms}

The cognitive and emotional deficits $[86,87 \cdot]$ arising as a consequence of repeated detoxification episodes may not only contribute to inflexible and uncontrolled impulsive behaviour but also to deficits in social interaction that lead to social isolation, in turn supporting further drinking behaviour.

Several studies have demonstrated that alcoholic patients generally show an inability to recognise emotions in others $[50,51,61]$ and we have found that such impairments may be more pronounced as the number of detoxification attempts increase [90]. Knowing that amygdala is the brain structure mostly related with processing of fear [54] including the recognition of fearful facial expressions [21], for our studies of emotional competence, we centred our research efforts in revealing the role of the amygdala and its connections in the impairments seen in alcoholic patients and binge drinkers [86,87•].

Imaging the brain of alcoholic patients during fear recognition in emotional facial expression (EFE) of fear [71], revealed reduced connectivity in alcoholic patients between insula, centrally implicated in filtering emotions, and prefrontal emotional regulatory sites with which insula interacts $[65,66,73,82]$. In particular, a reduced connectivity of insula with the anterior cingulate cortex and the obitofrontal cortex was seen in alcoholic patients with two or more detoxifications compared with either controls or patients with a single or no detoxification [71]. Such reduced connectivity might be expected to lead to general deficits in emotional processing (e.g., $[18,82])$. On the other hand, the reduced connectivity between amygdala and globus pallidus, found in the same population, may reflect a dysfunction in emotionally driven motivational outputs.

In contrast with the impaired connectivity we frequently find in patients with multiple detoxifications, increased connectivity was found in alcoholic patients between insula and a colliculus neuronal cluster. This region represents an important subcortical area for arousal mechanisms, mediating transfer of fear signals to the locus coeruleus [55] without the need for processing in cortical regions. For this reason, such a pathway may allow fearful responses without conscious appraisal of threat (see also [5]).

Increased connectivity was also found between amygdala and bed nucleus of stria terminalis (BNST). BNST is one of the key components of stress-induced relapse in animal models of drug use disorder [4] and finds a central role in Koob's hedonic "allostasis model of addiction" [47,48]. Since BNST activation is associated with continuous monitoring of potential threat [83], it has been suggested that increased connectivity between amygdala and BNST seen in alcoholic patients facilitates neural integration of these structures, allowing threatening stimuli to be more efficiently processed within the extended amygdala. In our studies, when patients were required to identify the emotion being expressed in faces (explicit fear recognition), the strength of connectivity between insula and areas involved in control of behaviour and regulation of emotion (inferior frontal cortex [IFC], frontal pole) was negatively correlated with the number of detoxifications and the control over drinking evaluated by a questionnaire (Impaired Control Questionnaire [ICQ]; [71••]). When, however, patients observed the faces without the requirement to identify the emotion (passive view of emotion), the number of detoxifications and ICQ correlated positively with connectivity between the amygdala and a large cluster of prefrontal cortex areas involved in attentional and executive processes (e.g., dorsolateral prefrontal cortex). Thus, it seems that during a passive view of threat stimuli, amygdala-related neural networks become more strongly integrated as severity of alcoholism increases. In contrast, when recognition and regulation of emotion is required, insula-related networks are less well integrated as severity of alcoholism increases. Again, we 
can speculate that increased connectivity in amygdala-related networks would lead to an increased emotional reactivity [19], whereas decreases in the network integrity of insula-related networks would lead to inappropriate analysis of the emotional input [10•].

Taking the data together, we can summarise that, in alcoholic patients, connectivity between insula and other cortical structures underlying a top-down control of emotional input is diminished, whilst connectivity between insula and subcortical regions (colliculus), as well as between amygdala and other subcortical regions (BNST) underlying a bottom-up emotional input, is exaggerated. Such changes in connectivity in alcoholic patients may contribute to altered anxiety reactions, and thus to increased risk of stress-induced relapse.

Social competence measured by EFE in alcoholic patients was found to correlate with gray matter volume in several frontal and prefrontal areas. Fear recognition, found impaired in alcoholic patients, in particular in those with multiple detoxifications [90] is negatively correlated with grey matter volume in IFG $(\mathrm{r}=0.36$, $p=0.006$; unpublished), the area supporting inhibitory control. It seems that perception of emotion from the fearful faces was not regulated to allow correct recognition, probably due to the deficient IFC. It is of interest that in a post-hoc analysis we found that the higher the trait anxiety ratings, the worse the recognition of fearful faces $(r=-358, p=0.005)$. It is possible that increased anxiety led to increased emotional input leading to greater insufficiency of inhibition by IFG. It is worth noting that IFG may have some specific role in fearful expression recognition (e.g., [77]).

\section{Conclusions}

The present review has introduced a model of withdrawal kindling and its consequences for relapse (see Fig. 1). Relapse to alcohol abuse remains a significant problem in the treatment of alcoholic patients, but an under-recognised problem is the consequences of detoxification itself for the ability of the patient with AUD to retain control of drinking. Since detoxification has deleterious consequences for the function of the multiple brain mechanisms that underlie emotional and cognitive competence, including the ability to control the impulse to drink, more attention needs to be paid to this area of research. The impairments seen in alcoholics in the ICT and in the EFE recognition tasks point to adverse effects of repeated detoxification on two functions crucial for successful abstinence (on the one hand making appropriate decisions to avoid risk, and on the other allowing for emotions to be perceived and expressed accurately in order to support a social network). The development of psychometric tasks modelling such deficits seen in patients may help in identifying detoxification-induced deficits, and in assessing potential treatments that may be helpful in restoring function. We have outlined a number of tests that address particular deficits found in patients with AUD. However, it remains to be investigated whether individuals with other drug use disorders, or other impulsive or compulsive disorders, may show similar deficits in these tasks, or whether such deficits are remediable by drug or behavioural therapies, including those that are currently available for treatment of AUD.

Acknowledgment The research of the authors described in this review was supported by the UK Medical Research Council.

\section{Compliance with Ethics Guidelines}

Conflict of Interest Theodora Duka and David N. Stephens declare that they have no conflict of interest.

Human and Animal Rights and Informed Consent This article reviews studies carried out by the authors on human and animal subjects. Studies on humans were carried out following ethical approval by the appropriate local institutional ethical review boards. Animals studies were performed in accordance with the UK Animals (Scientific procedures) Act 1986, following ethical review by the local ethical review committee.

\section{References}

Recently published papers of particular importance have been highlighted as:

- Of importance,

•- Of major importance

1.• Anderson BA, Laurent PA, Yantis S. Value-driven attentional capture. Proc Natl Acad Sci U S A. 2011;108:10367-71. The paper describes a unique attentional capture to stimuli that acquire value through associative learning. It proposes that this form of attentional capture may provide a useful model for investigating failures of cognitive control in clinical conditions in which the value of stimuli conflicts with behavioral goals (e.g., addiction).

2. Anton RF, O'Malley SS, Ciraulo DA, Cisler RA, Couper D, Donovan DM, et al. Combined pharmacotherapies and behavioral interventions for alcohol dependence: the COMBINE study: a randomized controlled trial. JAMA. 2006;295:2003-17.

3. APA. PsycINFO Database Record (c); 2012.

4. Aston-Jones G, Harris GC. Brain substrates for increased drug seeking during protracted withdrawal. Neuropharmacology. 2004;47 Suppl 1:167-79.

5. Baas JM, Milstein J, Donlevy M, Grillon C. Brainstem correlates of defensive states in humans. Biol Psychiatry. 2006;59:588-93.

6. Baker TB, Cannon DS. Potentiation of ethanol withdrawal by prior dependence. Psychopharmacology (Berlin). 1979;60:105-10.

7. Baumeister RF. Ego depletion and self-regulation failure: a resource model of self-control. Alcohol Clin Exp Res. 2003;27: 281-4.

8. Bechara A. Neurobiology of decision-making: risk and reward. Semin Clin Neuropsychiatry. 2001;6:205-16. 
9. Bechara A, Tranel D, Damasio H. Characterization of the decisionmaking deficit of patients with ventromedial prefrontal cortex lesions. Brain. 2000;123(Pt 11):2189-202.

10. Bechara A, Van Der Linden M. Decision-making and impulse control after frontal lobe injuries. Curr Opin Neurol. 2005;18: 734-9. The paper describes the role of ventromedial prefrontal cortex in decision making under emotional conflict.

11. Becker HC, Hale RL. Repeated episodes of ethanol withdrawal potentiate the severity of subsequent withdrawal seizures: an animal model of alcohol withdrawal "kindling". Alcohol Clin Exp Res. 1993;17:94-8.

12. Berns GS, McClure SM, Pagnoni G, Montague PR. Predictability modulates human brain response to reward. J Neurosci. 2001;21: 2793-8.

13. Bischoff-Grethe A, Hazeltine E, Bergren L, Ivry RB, Grafton ST. The influence of feedback valence in associative learning. Neuroimage. 2009;44:243-51.

14.•• Borlikova GG, Elbers NA, Stephens DN. Repeated withdrawal from ethanol spares contextual fear conditioning and spatial learning but impairs negative patterning and induces over-responding: evidence for effect on frontal cortical but not hippocampal function? Eur J Neurosci. 2006;24:205-16. The paper describes in animals an homologous incentive conflict task which was also impaired in animals having undergone multiple detoxifications.

15. Brewer JA, Worhunsky PD, Carroll KM, Rounsaville BJ, Potenza $\mathrm{MN}$. Pretreatment brain activation during stroop task is associated with outcomes in cocaine-dependent patients. Biol Psychiatry. 2008;64(11):998-1004.

16. Brown ME, Anton RF, Malcolm R, Ballenger JC. Alcohol detoxification and withdrawal seizures: clinical support for a kindling hypothesis. Biol Psychiatry. 1988;23:507-14.

17. Cox WM, Hogan LM, Kristian MR, Race JH. Alcohol attentional bias as a predictor of alcohol abusers' treatment outcome. Drug Alcohol Depend. 2002;68:237-43.

18. Craig AD. How do you feel? Interoception: the sense of the physiological condition of the body. Nat Rev Neurosci. 2002;3:655-66.

19. Davis M, Whalen PJ. The amygdala: vigilance and emotion. Mol Psychiatry. 2001;6:13-34.

20. De Bellis MD, Narasimhan A, Thatcher DL, Keshavan MS, Soloff P, Clark DB. Prefrontal cortex, thalamus, and cerebellar volumes in adolescents and young adults with adolescent-onset alcohol use disorders and comorbid mental disorders. Alcohol Clin Exp Res. 2005;29:1590-600.

21. Derntl B, Habel U, Windischberger C, Robinson S, Kryspin-Exner I, Gur RC, et al. General and specific responsiveness of the amygdala during explicit emotion recognition in females and males. BMC Neurosci. 2009;10:91.

22. Derryberry D, Rothbart MK. Reactive and effortful processes in the organization of temperament. Dev Psychopathol. 1997;9:633-52.

23. Dretsch MN, Tipples J. Working memory involved in predicting future outcomes based on past experiences. Brain Cogn. 2008;66: $83-90$.

24. Duka T, Gentry J, Malcolm R, Ripley TL, Borlikova G, Stephens $\mathrm{DN}$, et al. Consequences of multiple withdrawals from alcohol. Alcohol Clin Exp Res. 2004;28:233-46.

25. Duka T, Townshend JM, Collier K, Stephens DN. Kindling of withdrawal: a study of craving and anxiety after multiple detoxifications in alcoholic inpatients. Alcohol Clin Exp Res. 2002;26: 785-95.

26. Duka T, Townshend JM, Collier K, Stephens DN. Impairment in cognitive functions after multiple detoxifications in alcoholic inpatients. Alcohol Clin Exp Res. 2003;27:1563-72.

27.• Duka T, Trick L, Nikolaou K, Gray MA, Kempton MJ, Williams H, et al. Unique brain areas associated with abstinence control are damaged in multiply detoxified alcoholics. Biol Psychiatry. 2011;70:545-52. The paper describes the deficits in incentive conflict found in AUD patients with multiple detoxifications and identifies the areas of the brain underlying these deficits.

28. Elliott R, Agnew Z, Deakin JF. Medial orbitofrontal cortex codes relative rather than absolute value of financial rewards in humans. Eur J Neurosci. 2008;27:2213-8.

29. Ernst M, Paulus MP. Neurobiology of decision making: a selective review from a neurocognitive and clinical perspective. Biol Psychiatry. 2005;58:597-604.

30. Etkin A, Egner T, Peraza DM, Kandel ER, Hirsch J. Resolving emotional conflict: a role for the rostral anterior cingulate cortex in modulating activity in the amygdala. Neuron. 2006;51:871-82.

31. Everitt BJ, Dickinson A, Robbins TW. The neuropsychological basis of addictive behaviour. Brain Res Brain Res Rev. 2001;36: 129-38.

32. Field M, Cox WM. Attentional bias in addictive behaviors: a review of its development, causes, and consequences. Drug Alcohol Depend. 2008;97:1-20.

33. Finn PR. Motivation, working memory, and decision making: a cognitive-motivational theory of personality vulnerability to alcoholism. Behav Cogn Neurosci Rev. 2002;1:183-205.

34. Floden D, Stuss DT. Inhibitory control is slowed in patients with right superior medial frontal damage. J Cogn Neurosci. 2006;18: 1843-9.

35.• Garavan H, Weierstall K. The neurobiology of reward and cognitive control systems and their role in incentivizing health behavior. Prev Med. 2012;55:S17-23. The paper describes how frontal lobes regulate striatal reward-related processes and highlights the importance of prefrontal control systems for a successful recovery from addiction.

36. George DT, Nutt DJ, Dwyer BA, Linnoila M. Alcoholism and panic disorder: is the comorbidity more than coincidence? Acta Psychiatr Scand. 1990;81:97-107.

37. Goldberg II, Harel M, Malach R. When the brain loses its self: prefrontal inactivation during sensorimotor processing. Neuron. 2006;50:329-39.

38. Goldstein RZ, Volkow ND. Drug addiction and its underlying neurobiological basis: neuroimaging evidence for the involvement of the frontal cortex. Am J Psychiatry. 2002;159:1642-52.

39. Goldstein RZ, Woicik PA, Maloney T, Tomasi D, Alia-Klein N, Shan J, et al. Oral methylphenidate normalizes cingulate activity in cocaine addiction during a salient cognitive task. Proc Natl Acad Sci U S A. 2011;107:16667-72.

40. Hester R, Dixon V, Garavan H. A consistent attentional bias for drug-related material in active cocaine users across word and picture versions of the emotional Stroop task. Drug Alcohol Depend. 2006;81:251-7.

41. Hester R, Garavan H. Neural mechanisms underlying drug-related cue distraction in active cocaine users. Pharmacol Biochem Behav. 2009;93:270-7.

42. Hofmann W, Gschwendner T, Friese M, Wiers RW, Schmitt M. Working memory capacity and self-regulatory behavior: toward an individual differences perspective on behavior determination by automatic versus controlled processes. J Pers Soc Psychol. 2008;95:962-77.

43. Jang DP, Namkoong K, Kim JJ, Park S, Kim IY, Kim SI, et al. The relationship between brain morphometry and neuropsychological performance in alcohol dependence. Neurosci Lett. 2007;428:21-6.

44. Johan Franck J, Jayaram-Lindstr N. Pharmacotherapy for alcohol dependence: status of current treatment. Curr Opin Neurobiol. 2013;23:692-9.

45. Kalivas PW, Volkow ND. The neural basis of addiction: a pathology of motivation and choice. Am J Psychiatry. 2005; 162:1403-13.

46. Kim C, Johnson NF, Cilles SE, Gold BT. Common and distinct mechanisms of cognitive flexibility in prefrontal cortex. J Neurosci. 2011;31:4771-9. 
47. Koob GF. Alcoholism: allostasis and beyond. Alcohol Clin Exp Res. 2003;27:232-43.

48. Koob GF. Brain stress systems in the amygdala and addiction. Brain Res. 2009;1293:61-75.

49. Kornreich C, Blairy S, Philippot P, Dan B, Foisy M, Hess U, et al. Impaired emotional facial expression recognition in alcoholism compared with obsessive-compulsive disorder and normal controls. Psychiatry Res. 2001;102:235-48.

50. Kornreich C, Blairy S, Philippot P, Hess U, Noel X, Streel E, et al. Deficits in recognition of emotional facial expression are still present in alcoholics after mid- to long-term abstinence. J Stud Alcohol. 2001;62:533-42.

51. Kornreich C, Philippot P, Foisy ML, Blairy S, Raynaud E, Dan B, et al. Impaired emotional facial expression recognition is associated with interpersonal problems in alcoholism. Alcohol Alcohol. 2002;37:394-400.

52. Krystal JH, Webb E, Grillon C, Cooney N, Casal L, Morgan 3rd $\mathrm{CA}$, et al. Evidence of acoustic startle hyperreflexia in recently detoxified early onset male alcoholics: modulation by yohimbine and m-chlorophenylpiperazine (mCPP). Psychopharmacology (Berlin). 1997;131:207-15.

53. Lechtenberg R, Worner TM. Relative kindling effect of detoxification and non-detoxification admissions in alcoholics. Alcohol Alcohol. 1991;26:221-5.

54. LeDoux JE. Emotion circuits in the brain. Annu Rev Neurosci. 2000;23:155-84.

55. Liddell BJ, Brown KJ, Kemp AH, Barton MJ, Das P, Peduto A, et al. A direct brainstem-amygdala-cortical 'alarm' system for subliminal signals of fear. Neuroimage. 2005;24:235-43.

56. Loeber S, Duka T, Welzel H, Nakovics H, Heinz A, Flor H, et al. Impairment of cognitive abilities and decision making after chronic use of alcohol: the impact of multiple detoxifications. Alcohol Alcohol. 2009;44:372-81.

57. Luijten M, Veltman DJ, van den Brink W, Hester R, Field M, Smits $\mathrm{M}$, et al. Neurobiological substrate of smoking-related attentional bias. Neuroimage. 2011;54:2374-81.

58. Mann K, Bladstrom A, Torup L, Gual A, van den Brink W. Extending the treatment options in alcohol dependence: a randomized controlled study of as-needed nalmefene. Biol Psychiatry. 2013;73:706-13.

59. Mann K, Lehert P, Morgan MY. The efficacy of acamprosate in the maintenance of abstinence in alcohol-dependent individuals: results of a meta-analysis. Alcohol Clin Exp Res. 2004;28:51-63.

60. Mann T, Ward A. Attention, self-control, and health behaviors. Curr Dir Psychol Sci. 2007;16:280-3.

61. Maurage P, Campanella S, Philippot P, Charest I, Martin S, de Timary P. Impaired emotional facial expression decoding in alcoholism is also present for emotional prosody and body postures. Alcohol Alcohol. 2009;44:476-85.

62. McClure SM, York MK, Montague PR. The neural substrates of reward processing in humans: the modern role of FMRI. Neuroscientist. 2004;10:260-8.

63. McCown TJ, Breese GR. Multiple withdrawals from chronic ethanol "kindles" inferior collicular seizure activity: evidence for kindling of seizures associated with alcoholism. Alcohol Clin Exp Res. 1990;14:394-9.

64. Medina KL, Schweinsburg AD, Cohen-Zion M, Nagel BJ, Tapert SF. Effects of alcohol and combined marijuana and alcohol use during adolescence on hippocampal volume and asymmetry. Neurotoxicol Teratol. 2007;29:141-52.

65. Mesulam MM, Mufson EJ. Insula of the old world monkey. I. Architectonics in the insulo-orbito-temporal component of the paralimbic brain. J Comp Neurol. 1982;212:1-22.

66. Mesulam MM, Mufson EJ. Insula of the old world monkey. III: Efferent cortical output and comments on function. J Comp Neurol. $1982 ; 212: 38-52$.
67. Mitchell DG, Luo Q, Avny SB, Kasprzycki T, Gupta K, Chen G, et al. Adapting to dynamic stimulus-response values: differential contributions of inferior frontal, dorsomedial, and dorsolateral regions of prefrontal cortex to decision making. J Neurosci. 2009;29:10827-34.

68. Moselhy HF, Georgiou G, Kahn A. Frontal lobe changes in alcoholism: a review of the literature. Alcohol Alcohol. 2001;36:357-68.

69. Nagel BJ, Schweinsburg AD, Phan V, Tapert SF. Reduced hippocampal volume among adolescents with alcohol use disorders without psychiatric comorbidity. Psychiatry Res. 2005;139:181-90.

70. Nikolaou K, Field M, Critchley H, Duka T. Acute alcohol effects on attentional bias are mediated by subcortical areas associated with arousal and salience attribution. Neuropsychopharmacology. 2013;38:1365-73.

71.• O'Daly OG, Trick L, Scaife J, Marshall J, Ball D, Phillips ML, et al. Withdrawal-associated increases and decreases in functional neural connectivity associated with altered emotional regulation in alcoholism. Neuropsychopharmacology. 2012;37:2267-76. The paper describes the areas of the brain showing changes in activation when patients with AUD are presented with emotional facial expression of fear. More importantly shows neuronal connectivity differences when normal controls are compared with patients with AUD. These differences suggest a decreased connectivity between structures involved in top-down emotional control and an increased connectivity between structures involved in bottomup functions associated with arousal and stress sensitivity.

72. O'Doherty JP, Deichmann R, Critchley HD, Dolan RJ. Neural responses during anticipation of a primary taste reward. Neuron. 2002;33:815-26.

73. Ongur D, Ferry AT, Price JL. Architectonic subdivision of the human orbital and medial prefrontal cortex. J Comp Neurol. 2003;460:425-49.

74. Overstreet DH, Knapp DJ, Breese GR. Accentuated decrease in social interaction in rats subjected to repeated ethanol withdrawals. Alcohol Clin Exp Res. 2002;26:1259-68.

75. Picton TW, Stuss DT, Alexander MP, Shallice T, Binns MA, Gillingham S. Effects of focal frontal lesions on response inhibition. Cereb Cortex. 2007;17:826-38.

76. Pinel JP, Van Oot PH, Mucha RF. Intensification of the alcohol withdrawal syndrome by repeated brain stimulation. Nature. 1975;254:510-1.

77. Rahko J, Paakki JJ, Starck T, Nikkinen J, Remes J, Hurtig $\mathrm{T}$, et al. Functional mapping of dynamic happy and fearful facial expression processing in adolescents. Brain Imaging Behav. 2010;4:164-76.

78. Rehm J, Shield KD, Gmel G, Rehm MX, Frick U. Modeling the impact of alcohol dependence on mortality burden and the effect of available treatment interventions in the European Union. Eur Neuropsychopharmacol. 2013;23:89-97.

79. Rescorla RA. "Configural" conditioning in discrete-trial bar pressing. J Comp Physiol Psychol. 1972;79:307-17.

80. Ripley TL, O'Shea M, Stephens DN. Repeated withdrawal from ethanol impairs acquisition but not expression of conditioned fear. Eur J Neurosci. 2003;18(2):441-8.

81. Robinson TE, Berridge KC. The neural basis of drug craving: an incentive-sensitization theory of addiction. Brain Res Brain Res Rev. 1993;18:247-91.

82. Singer T, Critchley HD, Preuschoff K. A common role of insula in feelings, empathy and uncertainty. Trends Cogn Sci. 2009;13: 334-40.

83. Somerville LH, Whalen PJ, Kelley WM. Human bed nucleus of the stria terminalis indexes hypervigilant threat monitoring. Biol Psychiatry. 2010;68:416-24.

84. Squeglia LM, Schweinsburg AD, Pulido C, Tapert SF. Adolescent binge drinking linked to abnormal spatial working memory brain 
activation: differential gender effects. Alcohol Clin Exp Res. 2011;35:1831-41.

85. Stephens DN, Brown G, Duka T, Ripley TL. Impaired fear conditioning but enhanced seizure sensitivity in rats given repeated experience of withdrawal from alcohol. Eur J Neurosci. 2001;14:202331.

86. Stephens DN, Duka T. Review. Cognitive and emotional consequences of binge drinking: role of amygdala and prefrontal cortex. Philos Trans R Soc Lond B Biol Sci. 2008;363:3169-79.

87. Stephens DN, Ripley TL, Borlikova G, Schubert M, Albrecht D, Hogarth L, et al. Repeated ethanol exposure and withdrawal impairs human fear conditioning and depresses long-term potentiation in rat amygdala and hippocampus. Biol Psychiatry. 2005;58:392-400. The paper describes data supporting a model of increased neuronal activation accompanied by decreased plasticity due to synaptic saturation blocking further plasticity and leading to associative learning impairments.
88. Tobler PN, O'Doherty JP, Dolan RJ, Schultz W. Human neural learning depends on reward prediction errors in the blocking paradigm. J Neurophysiol. 2006;95:301-10.

89. Townshend JM, Duka T. Attentional bias associated with alcohol cues: differences between heavy and occasional social drinkers. Psychopharmacology (Berl). 2001;157:67-74.

90. Townshend JM, Duka T. Mixed emotions: alcoholics' impairments in the recognition of specific emotional facial expressions. Neuropsychologia. 2003;41:773-82.

91. Townshend JM, Duka T. Binge drinking, cognitive performance and mood in a population of young social drinkers. Alcohol Clin Exp Res. 2005;29:317-25.

92. Tucker DM, Luu P, Pribram KH. Social and emotional selfregulation. Ann N Y Acad Sci. 1995;769:213-39.

93. Volkow ND, Fowler JS, Wang GJ. The addicted human brain: insights from imaging studies. J Clin Invest. 2003;111:1444-51.

94. Woodbury CB. The learning of stimulus patterns by dogs. J Comp Physiol Psychol. 1943;35:29-40. 